\begin{tabular}{|c|l|}
\hline Title & The drift velocity vector of electron swarms in crossed electric and magnetic fields \\
\hline Author(s) & Sugawara, Hirotake; Y ahata, Takashi; Oda, A kinori; Sakai, Y osuke \\
\hline Citation & Journal of Physics D: A pplied Physics, 33(10), 1191-1196 \\
\hline https://doi.org/10.1088/0022-3727/33/10/309 \\
\hline Issue Date & 2000-05-21 \\
\hline Doc URL & http://hdl.handle.net/2115/1468 \\
\hline Rights & Copyright $\odot$ 2000 IOP Publishing Ltd. \\
\hline Type & article (author version) \\
\hline Note(URL) & http://www.iop.org/ \\
\hline Information & \\
\hline
\end{tabular}

Instructions for use 


\title{
The drift velocity vector of electron swarms in crossed electric and magnetic fields $\dagger$
}

\author{
Hirotake Sugawara, Takashi Yahata, Akinori Oda and Yosuke Sakai \\ Division of Electronics and Information Engineering, Hokkaido University, Sapporo \\ 060-8628 Japan
}

\begin{abstract}
We calculated the drift velocity vector $\boldsymbol{W}$ of electron swarms in crossed electric and magnetic fields using a Monte Carlo method. Values of $\boldsymbol{W}$ in $\mathrm{F}_{2}, \mathrm{CF}_{4}$ and constant-collision-frequency $(\mathrm{CCF})$ model gases at various values of the reduced electric and magnetic fields, $E / N$ and $B / N$ ( $N$ is the gas molecule number density), were illustrated as vector loci in velocity space. Under a CCF condition, $\boldsymbol{W}$ draws right semicircular loci with varying $B$ and $N$ at a constant $E$ when the number of electrons is conservative. These semicircular loci can be a reference to characterize the $\boldsymbol{W}$ behaviour in real gases. We presented an analytical derivation of $\boldsymbol{W}$ in the CCF condition, extending its expression to that in the presence of ionization and attachment. Features of the vector loci in $\mathrm{F}_{2}$ and $\mathrm{CF}_{4}$ were discussed.
\end{abstract}

PACS numbers: 52.80.-s Electric discharges

Short title: Electron drift velocity in $\boldsymbol{E} \times \boldsymbol{B}$ fields

† Published source: Journal of Physics D: Applied Physics, Vol. 33, No. 10, pp. 1191-1196 (2000) 


\section{Introduction}

The electron swarm development in crossed electric and magnetic fields $(\boldsymbol{E} \times \boldsymbol{B}$ field $)$ is a fundamental process in magnetized plasmas such as inductively coupled plasmas and magnetron plasmas. Under a recent tendency where simulations of such plasmas have been performed in three-dimensional models (e.g. Hopwood 1992, Nanbu and Kondo 1997, Shon et al 1999), it is important to consider the direction-dependent electron transport in $\boldsymbol{E} \times \boldsymbol{B}$ fields as a primary factor to govern the plasma behaviour.

A survey of concepts quantifying the electron transport in $\boldsymbol{E} \times \boldsymbol{B}$ fields was presented by Heylen (1980), and a lot of progressive work has previously been reported. Dincer and Gokmen (1992) measured electrical breakdown properties of $\mathrm{SF}_{6}$, and subsequently Dincer (1993) and Milsom (1993) calculated electron transport coefficients in $\mathrm{SF}_{6}$ employing a Monte Carlo method. Shimura and Makabe (1993) analyzed the electron velocity distribution in Ar using a Boltzmann equation technique. Pekker (1995) and Cramer (1997) performed one-dimensional analyses of Ar plasmas between parallel-plane electrodes. Nakamura et al (1999) presented electron swarm parameters in $\mathrm{Ar}$ and $\mathrm{CH}_{4}$ calculated with a Monte Carlo method, being conscious of inductive plasma sources.

In this paper, we calculate the drift velocity vector $\boldsymbol{W}$ of electron swarms in $\mathrm{F}_{2}$ and $\mathrm{CF}_{4}$ in $\boldsymbol{E} \times \boldsymbol{B}$ fields using a Monte Carlo method. The dependence of $\boldsymbol{W}$ on the magnetic field and the gas molecule number density (or the gas pressure) was depicted as vector loci in velocity space. In order to characterize the behaviour of $\boldsymbol{W}$ in real gases, we analyse $\boldsymbol{W}$ under a constant-collision-frequency (CCF) condition as a reference. The vector loci under a CCF condition are right semicircles when the number of electrons is conservative, which reflects the essential $\boldsymbol{W}$ behaviour in $\boldsymbol{E} \times \boldsymbol{B}$ fields.

CCF conditions have often been assumed to simplify the calculation in theoretical studies. Blevin and Haydon (1958) estimated the average electron collision frequency in $\mathrm{H}_{2}$ from experimental data of the electron drift velocity. Heylen and Bunting (1969) evaluated this estimation quantitatively. Bunting and Heylen (1971) and Heylen and 
Dargon (1973) performed similar evaluations for $\mathrm{N}_{2}, \mathrm{O}_{2}$ and air as well. White et al (1997) employed a CCF condition in benchmark simulations for various numerical methods of swarm analyses in $\boldsymbol{E} \times \boldsymbol{B}$ fields. White et al (1999) performed a Boltzmann equation analysis in a CCF model in $\boldsymbol{E} \times \boldsymbol{B}$ fields crossed at arbitrary angles as well.

We show a detailed derivation of the analytical expression of $\boldsymbol{W}$ in a CCF condition, now for the first time taking account of the effects of ionization and attachment on $\boldsymbol{W}$. Features of the vector loci in $\mathrm{F}_{2}$ and $\mathrm{CF}_{4}$ were discussed.

\section{Derivation of the drift velocity vector}

An electron undergoes free flight and collision (scattering) processes alternately. The former is described as single electron motion based on the motion equation. The latter quantified by the collision frequency determines the start and end of a free flight. We define the drift velocity vector $\boldsymbol{W}$ of an electron swarm in $\boldsymbol{E} \times \boldsymbol{B}$ fields as being the average electron velocity vector over the swarm. We must consider the change in the electron population when calculating $\boldsymbol{W}$ in the presence of ionization and/or attachment.

\subsection{Single electron motion}

Uniform dc electric and magnetic fields are applied to the $-z$ and $+y$ directions in real space $(x, y, z)$ as $\boldsymbol{E}=(0,0,-E)$ and $\boldsymbol{B}=(0, B, 0)$ (see also figure 1). With an initial electron velocity $\boldsymbol{v}^{\prime}=\left(v_{x}^{\prime}, v_{y}^{\prime}, v_{z}^{\prime}\right)$ at time $t^{\prime}$, the motion equation, $m(\mathrm{~d} \boldsymbol{v} / \mathrm{d} t)=-e \boldsymbol{v} \times \boldsymbol{B}-e \boldsymbol{E}$, yields the following solution for the electron velocity $\boldsymbol{v}(t)=\left(v_{x}, v_{y}, v_{z}\right)$ :

$$
\begin{aligned}
& v_{x}=E / B+v_{z}^{\prime} \sin \omega\left(t-t^{\prime}\right)+\left(v_{x}^{\prime}-E / B\right) \cos \omega\left(t-t^{\prime}\right) \\
& v_{y}=v_{y}^{\prime} \\
& v_{z}=v_{z}^{\prime} \cos \omega\left(t-t^{\prime}\right)-\left(v_{x}^{\prime}-E / B\right) \sin \omega\left(t-t^{\prime}\right)
\end{aligned}
$$


where $e$ and $m$ are the electron charge and mass, and $\omega=e B / m$. Elimination of a factor $\left(t-t^{\prime}\right)$ from equations (1) and (3) gives the following circular locus of an electron:

$$
\left(v_{x}-E / B\right)^{2}+v_{z}^{2}=\left(v_{x}^{\prime}-E / B\right)^{2}+v_{z}^{\prime 2}
$$

The electrons orbit the center $\mathrm{C}$ at $\left(v_{x}, v_{z}\right)=(E / B, 0)$ with a constant angular velocity $\omega$ during free flight (see figure 2).

\subsection{Collision and scattering}

The present model assumes the following conditions. The total collision frequency $\nu$ for an electron is constant, being independent of the electron energy (CCF condition). Scattering due to collision is isotropic in laboratory system, and the momentum loss at an elastic collision is negligible. The initial velocity distribution of secondary electrons produced by ionization is also isotropic. The ionization and attachment frequencies, $\nu_{\mathrm{i}}$ and $\nu_{\mathrm{a}}$, are constant in equilibrium, thus the electron population varies exponentially with time.

After scattering, most electrons start their next free flights with non-zero initial velocities $\left(\boldsymbol{v}^{\prime} \neq 0\right)$. Let us call such electrons warm-start electrons. However, Blevin and Haydon (1958) and Heylen (1980) assumed $\boldsymbol{v}^{\prime}=0$ for all the electrons (i.e. cold-start electrons) in their derivations for $\boldsymbol{W}$. For this treatment, Blevin and Haydon (1958) noted that an electron loses all its momentum in any specified direction on the average in a collision; they regarded the condition $\left\langle\boldsymbol{v}^{\prime}\right\rangle=0$ as if $\boldsymbol{v}^{\prime}=0$. Here, it was unconfirmed in the literature above whether the motion of a cold-start electron is equivalent to the average motion of the warm-start electrons, not only at the start but also throughout the flight even in the presence of collisions. We clarify this point in the following subsection.

\subsection{Free flight of electron squad}

In order to formulate the averaging scheme for $\boldsymbol{W}$, let us define an electron squad, a subset of the entire electron swarm, as being electrons starting free flight together at 
coincident collisions. The electron velocity distribution $f_{\mathrm{s}}\left(\boldsymbol{v}, t^{\prime}, t\right)$, the average velocity $\boldsymbol{v}_{\mathrm{s}}\left(t^{\prime}, t\right)$ and the electron population $\mathrm{d} n_{\mathrm{s}}\left(t^{\prime}, t\right)$ of a squad satisfy

$$
\begin{aligned}
\mathrm{d} n_{\mathrm{s}}\left(t^{\prime}, t\right) & =\int f_{\mathrm{s}}\left(\boldsymbol{v}, t^{\prime}, t\right) \mathrm{d} \boldsymbol{v} \\
\boldsymbol{v}_{\mathrm{s}}\left(t^{\prime}, t\right) & =\frac{\int \boldsymbol{v} f_{\mathrm{s}}\left(\boldsymbol{v}, t^{\prime}, t\right) \mathrm{d} \boldsymbol{v}}{\int f_{\mathrm{s}}\left(\boldsymbol{v}, t^{\prime}, t\right) \mathrm{d} \boldsymbol{v}}
\end{aligned}
$$

where $t^{\prime}$ and $t$ refer to times of birth and observation of the squad. The electrons scattered at collisions drop out of the squad and they form new squads together with dropouts from the other squads. Thus $\mathrm{d} n_{\mathrm{s}}\left(t^{\prime}, t\right)$ decreases monotonously with $t$.

For the isotropic scattering, $f_{\mathrm{s}}$ at $t=t^{\prime}$ is isotropic around $\boldsymbol{v}=0$, thus $\boldsymbol{v}_{\mathrm{s}}\left(t^{\prime}, t^{\prime}\right)=0$. A cold-start electron in the squad is at the center of the distribution $f_{\mathrm{s}}$ at this moment. The squad would simply rotate around $\mathrm{C}$ with the angular velocity $\omega$ during collisionless flight, therefore the electron formation in $f_{\mathrm{s}}$ is unchanged (see figure 2 ). The cold-start electron in the squad is always at the center of $f_{\mathrm{s}}$, thus its velocity is identical to $\boldsymbol{v}_{\mathrm{s}}\left(t^{\prime}, t\right)$. This situation also applies to the collisional case, because the electron dropout away from the squad due to collisions occurs uniformly in $f_{\mathrm{s}}$ as a result of the CCF condition. The value of $\mathrm{d} n_{\mathrm{s}}$ decays exponentially with $t$ keeping $f_{\mathrm{s}}$ isotropic around the cold-start electron (see figure 3 ). Therefore, although $f_{\mathrm{s}}$ is unknown, we can conclude that a coldstart electron represents the average motion of a squad without loss of generality in the CCF condition. Note here that the change of the electron population due to ionization and attachment does not affect this conclusion because the isotropy of $f_{\mathrm{s}}$ is kept in the collision processes under the CCF condition.

\subsection{Averaging over the swarm}

The drift velocity $\boldsymbol{W}$ of the entire electron swarm is obtained by integrating $\boldsymbol{v}_{\mathrm{s}}\left(t^{\prime}, t\right)$ over $t^{\prime} \in(-\infty, t]$, i.e. over all the squads born before observation, with the weight of $\mathrm{d} n_{\mathrm{s}}\left(t^{\prime}, t\right)$.

In equilibrium, the electron population of the entire swarm, $n$, is an exponential function given as $n(t)=n(0) \exp \left[\left(\nu_{\mathrm{i}}-\nu_{\mathrm{a}}\right) t\right]$. The initial value of $\mathrm{d} n_{\mathrm{s}}$ at $t=t^{\prime}$ is 
the number of scattered electrons in the entire swarm in a short interval $\mathrm{d} t^{\prime}$. The number of collisions during $\mathrm{d} t^{\prime}$ is $n\left(t^{\prime}\right) \nu \mathrm{d} t^{\prime}$, and taking account of lost electrons due to attachment and secondary electrons produced by ionization, we obtain $\mathrm{d} n_{\mathrm{s}}\left(t^{\prime}, t^{\prime}\right)=$ $n\left(t^{\prime}\right)\left(\nu+\nu_{\mathrm{i}}-\nu_{\mathrm{a}}\right) \mathrm{d} t^{\prime}$. For the dropout of scattered electrons, $\mathrm{d} n_{\mathrm{s}}$ decays exponentially as $\mathrm{d} n_{\mathrm{s}}\left(t^{\prime}, t\right)=\mathrm{d} n_{\mathrm{s}}\left(t^{\prime}, t^{\prime}\right) \exp \left[-\nu\left(t-t^{\prime}\right)\right]$. In consequence, we obtain $\mathrm{d} n_{\mathrm{s}}\left(t^{\prime}, t\right)=$ $n\left(t^{\prime}\right)\left(\nu+\nu_{\mathrm{i}}-\nu_{\mathrm{a}}\right) \exp \left[-\nu\left(t-t^{\prime}\right)\right] \mathrm{d} t^{\prime}$.

The two non-zero components of $\boldsymbol{W}, W_{E}$ and $W_{E \times B}$, are the average values of $v_{z}$ and $v_{x}$ in equations (1) and (3) under the cold-start condition $\boldsymbol{v}^{\prime}=0$. We obtain

$$
\begin{aligned}
\boldsymbol{W} & =\frac{1}{n(t)} \int_{-\infty}^{t} \boldsymbol{v}_{\mathrm{s}}\left(t^{\prime}, t\right) \mathrm{d} n_{\mathrm{s}}\left(t^{\prime}, t\right)=\int_{-\infty}^{t} \boldsymbol{v}\left(t^{\prime}, t\right) \nu^{\prime} \exp \left[-\nu^{\prime}\left(t-t^{\prime}\right)\right] \mathrm{d} t^{\prime} \\
W_{E} & =\left\langle v_{z}\right\rangle=\int_{0}^{\infty} \nu^{\prime} \exp \left(-\nu^{\prime} \tau\right) \frac{E}{B} \sin \omega \tau \mathrm{d} \tau=\frac{E}{B} \frac{\nu^{\prime} \omega}{\nu^{\prime 2}+\omega^{2}} \\
W_{E \times B} & =\left\langle v_{x}\right\rangle=\int_{0}^{\infty} \nu^{\prime} \exp \left(-\nu^{\prime} \tau\right) \frac{E}{B}(1-\cos \omega \tau) \mathrm{d} \tau=\frac{E}{B} \frac{\omega^{2}}{\nu^{\prime 2}+\omega^{2}}
\end{aligned}
$$

where $\tau=t-t^{\prime}$ is the free flight time up to observation and $\nu^{\prime}=\nu+\nu_{\mathrm{i}}-\nu_{\mathrm{a}}$ is the electron-population-modified total collision frequency.

\section{Properties of the drift velocity vector}

Equations (8) and (9) are different from the conventional expressions only in the factor $\nu^{\prime}$ instead of $\nu$. The present expressions are identical to the conventional ones when $\nu_{\mathrm{i}}=\nu_{\mathrm{a}}$. Quantities derived from $\boldsymbol{W}$ are extended to those in the presence of ionization and attachment by simply replacing $\nu$ with $\nu^{\prime}$; for example the Hall deflection angle $\theta$ satisfies $\tan \theta=W_{E \times B} / W_{E}=\omega / \nu^{\prime}$ instead of the conventional expression $\omega / \nu$.

Parameters $E$ and $B$ are externally controllable, and $\nu$ is proportional to the gas molecule number density $N$ (or the gas pressure $p$ ). The vector loci of $\boldsymbol{W}$ for varying $\nu^{\prime}$ under a constant $E / B$ and for varying $B$ under a constant $E / \nu^{\prime}$ are obtained by eliminating each of $\nu^{\prime}$ and $B$ from equations (8) and (9):

$$
\begin{aligned}
& W_{E}^{2}+\left(W_{E \times B}-\frac{1}{2} \frac{E}{B}\right)^{2}=\left(\frac{1}{2} \frac{E}{B}\right)^{2} \\
& \left(W_{E}-\frac{e E}{2 m \nu^{\prime}}\right)^{2}+W_{E \times B}^{2}=\left(\frac{e E}{2 m \nu^{\prime}}\right)^{2} .
\end{aligned}
$$


These are semicircles in parameter space $\left(W_{E \times B}, W_{E}\right)$ when $E / B$ and $E / \nu^{\prime}$ are constant (see figure 4). Here, it is not always easy to keep $\nu^{\prime}$ constant under varying $B$. The terms $\nu_{\mathrm{i}}$ and $\nu_{\mathrm{a}}$ included in $\nu^{\prime}$ may vary with $B$ through the change of the electron energy distribution. However, in the absence of ionization and attachment (electronconservative) under the CCF condition, constant $E / \nu^{\prime}(=E / \nu)$ is achievable by keeping $E / N$ constant.

Because of the geometrically simple shape, the semicircular vector loci can be a reference when characterizing the $\boldsymbol{W}$ behaviour in real gases. This visual expression of $\boldsymbol{W}$ would be informative for the control of $\boldsymbol{W}$ and design of plasma equipment. We present examples of the vector loci in model and real gases in the following section.

Another property of $\boldsymbol{W}$ in the electron-conservative CCF condition is that the plots of $W_{E}$ versus $\ln (\nu / \omega)$ for varying $\nu$ (or $N$ ) under constant $E / B$ and $W_{E \times B}$ versus $\ln (\nu / \omega)$ for varying $B$ (or $\omega)$ under constant $E / \nu(E / N)$ are symmetric in $\nu / \omega=1$ as we can write

$$
\begin{aligned}
W_{E} & =\frac{E}{B} \frac{\nu / \omega}{1+(\nu / \omega)^{2}}=\frac{E}{B} \frac{\omega / \nu}{1+(\omega / \nu)^{2}} \\
W_{E \times B} & =\frac{e E}{m \nu} \frac{\nu / \omega}{1+(\nu / \omega)^{2}}=\frac{e E}{m \nu} \frac{\omega / \nu}{1+(\omega / \nu)^{2}} .
\end{aligned}
$$

$W_{E}$ and $W_{E \times B}$ take their maxima, $E /(2 B)$ and $e E /(2 m \nu)$, at $\nu / \omega=1$.

The properties of $\boldsymbol{W}$ shown here typify qualitative tendencies of $\boldsymbol{W}$ in real gases.

\section{Numerical calculation method}

We calculated $\boldsymbol{W}$ in CCF model gases, $\mathrm{F}_{2}$ and $\mathrm{CF}_{4}$ at various values of $B / N$ and $E / N$ using a Monte Carlo method. The number of electrons sampled in each equilibrium was $10^{6}$ or more. Values of $\boldsymbol{W}$ at $B=0$ in the real gases were obtained from a Boltzmann equation analysis. The $\boldsymbol{E} \times \boldsymbol{B}$ drift velocity, $E / B$, was also evaluated to show the collisionless case $(\nu=0)$.

For efficient Monte Carlo performance, we adopted the stochastic choice of free flight time (Skullerud 1968). This method introduces an imaginary collision cross 
section $q_{\text {null }}(\geq 0)$ associating with no scattering (null collision) so that we obtain $N\left[q_{\mathrm{t}}(v)+q_{\text {null }}(v)\right] v=\nu(\mathrm{CCF})$ in a range of $v=|\boldsymbol{v}|$ of interest, where $q_{\mathrm{t}}$ is the total cross section of real collisions. The probability $P(\tau)=\exp \left[-\int_{0}^{\tau} N q_{\mathrm{t}}(v(t)) v(t) \mathrm{d} t\right]$ of a free flight time greater than $\tau$ is simplified as $P(\tau)=\exp (-\nu \tau)$. A free flight time $\tau$ is chosen as $\tau=\nu^{-1} \ln (1-\xi)$ by a uniformly distributed random number $\xi \in[0,1)$, and the kind of collisional event is selected in proportion to each collision cross section at $v(\tau)$.

Because a null collision interrupts a free flight for nothing and requires a load for the calculation of electron trajectory, a way to save the computational time is to set $\nu$ as low as possible but not violating $q_{\text {null }} \geq 0$. Providing a single $\nu$ value for a simulation, such $\nu$ would be given as $\nu=\left[N q_{\mathrm{t}}(v) v\right]_{\max }$. Moreover, we can refer to lower $\nu$ based on a fact that the $|\boldsymbol{v}|$ profile of an electron gyrating in an $\boldsymbol{E} \times \boldsymbol{B}$ field is periodic and within a finite range $\left[v_{\min }, v_{\max }\right]$. Here, from equations $(1)-(3)$ at $v_{z}=0, v_{\max }$ and $v_{\min }$ are given as

$$
\begin{aligned}
& v_{\max }^{2}=\left\{E / B+\left[\left(v_{x}^{\prime}-E / B\right)^{2}+v_{z}^{\prime 2}\right]^{1 / 2}\right\}^{2}+v_{y}^{\prime 2} \\
& v_{\min }^{2}=\left\{E / B-\left[\left(v_{x}^{\prime}-E / B\right)^{2}+v_{z}^{\prime 2}\right]^{1 / 2}\right\}^{2}+v_{y}^{\prime 2} .
\end{aligned}
$$

With a data table to find the optimum $\nu$ value for given $v_{\min }$ and $v_{\max }$, the computational time of a Monte Carlo simulation in $\mathrm{CF}_{4}$ at low $E / N$ and high $B / N$ values, at which electrons are concentrated in a low $v$ range around the Ramsauer-Townsend minimum, was less than $1 / 20$ of that of a Monte Carlo simulation with a single fixed $\nu$ value.

\section{Results and discussion}

\subsection{Constant-collision-frequency model gases}

We assumed three CCF model gases; case 1, an ionization-dominated model $\left(\nu_{\mathrm{i}}>\nu_{\mathrm{a}}\right)$; case 2 , an electron-conservative model $\left(\nu_{\mathrm{i}}=\nu_{\mathrm{a}}=0\right)$; and case 3 , an attachmentdominated model $\left(\nu_{\mathrm{i}}<\nu_{\mathrm{a}}\right)$. Their cross sections were assigned by table 1 and the 
following definitions (see also figure 5):

$$
\begin{aligned}
& q_{\mathrm{t}}(\epsilon)=q_{1}(\epsilon)+q_{2}(\epsilon)+q_{3}(\epsilon)+q_{4}(\epsilon)=\nu_{1}\left(N_{1} v_{1} \sqrt{\epsilon}\right)^{-1} \\
& q_{2}(\epsilon)=0.9 \times q_{\mathrm{t}}(\epsilon)\{1+\exp [30(0.1-\epsilon)]\}^{-1}\{1+\exp [10(\epsilon-1.0)]\}^{-1} \\
& q_{3}(\epsilon)=0.2 \times q_{\mathrm{t}}(\epsilon) \sqrt{\max \left(1-[\max (3.0-\epsilon, 0)]^{2}, 0\right)} \\
& q_{4}(\epsilon)=0.7 \times q_{\mathrm{t}}(\epsilon) \sqrt{\max \left(1-[\max (5.0-\epsilon, 0)]^{2}, 0\right)} .
\end{aligned}
$$

Here, $\epsilon$ is the electron energy in $\mathrm{eV}$, and $v_{1}$ is the electron velocity associating with $1 \mathrm{eV}$. We set the total collision frequency $\nu_{1}$ to be $3.0 \times 10^{9} \mathrm{~s}^{-1}$ at the gas pressure $p_{0}=133$ $\mathrm{Pa}(1$ Torr $)$ at $0{ }^{\circ} \mathrm{C}$. The gas molecule number density $N_{1}$ at this $p_{0}$ is $3.54 \times 10^{16} \mathrm{~cm}^{-3}$. $E$ was fixed at $15 \mathrm{Vcm}^{-1}, B=1.8-9.0 \mathrm{mT}$ and $p_{0}=13.3-66.5 \mathrm{~Pa}$.

Table 1. Assignment of the electron collision cross sections of constant-collisionfrequency model gases; $q_{\mathrm{m}}$, momentum transfer; $q_{\mathrm{a}}$, attachment; $q_{\mathrm{e}}$, excitation (threshold $2 \mathrm{eV}$ ); and $q_{\mathrm{i}}$, ionization (threshold $4 \mathrm{eV}$ ).

\begin{tabular}{lllll}
\hline & $q_{\mathrm{m}}$ & $q_{\mathrm{a}}$ & $q_{\mathrm{e}}$ & $q_{\mathrm{i}}$ \\
\hline Case $1\left(\nu_{\mathrm{i}}>\nu_{\mathrm{a}}\right)$ & $q_{1}+q_{2}$ & 0 & $q_{3}$ & $q_{4}$ \\
Case $2\left(\nu_{\mathrm{i}}=\nu_{\mathrm{a}}=0\right)$ & $q_{1}+q_{2}$ & 0 & $q_{3}+q_{4}$ & 0 \\
Case 3 $\left(\nu_{\mathrm{i}}<\nu_{\mathrm{a}}\right)$ & $q_{1}$ & $q_{2}$ & $q_{3}$ & $q_{4}$ \\
\hline
\end{tabular}

$\boldsymbol{W}$ is depicted in figure 6. The intersections of the semicircles drawn based on equations (10) and (11) are the theoretical prediction for $\boldsymbol{W}$ in case 2. The results of case 2 indicated by crossed squares are right at the intersections, which verifies the derivation of $\boldsymbol{W}$. In case $1, \boldsymbol{W}$ shifts towards the origin along the semicircles of equation (10). Ionization supplies isotropic secondary electrons, which reduces the directivity of the swarm by increasing $\nu^{\prime}$. Attachment in case 3 decreases $\nu^{\prime}$ by excluding the electrons captured by gas molecules from the swarm population, which appears as the shift in opposite way to case 1 . It is noted that $\boldsymbol{W}$ does not deviate from the locus represented by equation (10) under a constant $\nu$ even in the presence of ionization and attachment. 


\section{2. $F_{2}$}

$\mathrm{F}_{2}$ is an example of real gases that satisfy a CCF condition to some extent, i.e. the total collision frequency does not change significantly under the conditions examined here. The set of the electron collision cross sections of $\mathrm{F}_{2}$ taken from Hayashi and Nimura (1983) is shown in figure 7. The approximate CCF feature is probably due to the gradient $\mathrm{d} \lg q_{\mathrm{t}} / \mathrm{d} \lg \epsilon$ close to $-\frac{1}{2}$ in a range from a few electronvolts to tens electronvolts around which a major part of the electron energy distribution lies.

Figure 8 shows $\boldsymbol{W}$ in $\mathrm{F}_{2}$ at $E=60 \mathrm{Vcm}^{-1}, B=5-30 \mathrm{mT}$ and $p_{0}=13.3-79.8$ $\mathrm{Pa}$. Open and full squares indicate whether the effective ionization frequency, $\nu_{\mathrm{i}}-\nu_{\mathrm{a}}$, is positive (ionization-dominated) or negative (attachment-dominated), respectively. With varying $p_{0}$ under constant $B, \boldsymbol{W}$ draws fine semicircular loci. On the other hand, for changing $B$ under constant $p_{0}, \boldsymbol{W}$ deviates from the guiding semicircles inwards when ionization-dominated and outwards when attachment-dominated as discussed before. However, $\nu_{\mathrm{i}}$ and $\nu_{\mathrm{a}}$ are too small (only a few percents relative to $\nu$ ) to induce such an observable deviation. The deviation is attributed rather to the fact that the collision frequency of each electron is actually energy-dependent although the macroscopic collision frequency which is the integrated value over the swarm seems constant.

Dincer (1993) showed that the ionization coefficient in $\mathrm{SF}_{6}$ is a function of the effective reduced electric field $(E / N)_{\mathrm{eff}}=(E / N) \cos \theta$, where $\theta$ is the Hall angle. A similar situation is found also in $\mathrm{F}_{2}$. When we put $(E / \nu) \cos \theta=c$ assuming $\nu \propto N$, we obtain $W_{E}^{2}+W_{E \times B}^{2}=|\boldsymbol{W}|=(e c / m)^{2}$ which represents a circle of the center $(0,0)$ in parameter space $\left(W_{E \times B}, W_{E}\right)$. In figure 8 , the border between the regions of $\nu_{\mathrm{i}}>\nu_{\mathrm{a}}$ $(\square)$ and $\nu_{\mathrm{i}}<\nu_{\mathrm{a}}(\mathbf{\square})$ could be found around the broken quadrant.

\section{3. $C F_{4}$}

Figure 9 shows the set of the electron collision cross sections of $\mathrm{CF}_{4}$ taken from Bordage et al (1999). It is characteristic that $\mathrm{CF}_{4}$ has Ramsauer-Townsend minimum, and $\nu$ 
decreases significantly at low $E / N$ values. The calculation conditions for $\mathrm{CF}_{4}$ were $E=60 \mathrm{Vcm}^{-1}, B=5-70 \mathrm{mT}$ and $p_{0}=13.3-399 \mathrm{~Pa}$.

Figure 10 shows the $\boldsymbol{W}$ profile. As a general tendency, the Hall angle $\theta$ increases with $B$, which affects $(E / N)_{\text {eff }}$ through a factor $\cos \theta$. Then, low values of $\nu$ appear under high $B$, which results in the increase in $W_{E \times B}$ as expected from equation (9). At high gas pressures (i.e. at low $E / N$ values), the locus flattens significantly. In particular, a part of the curve for $399 \mathrm{~Pa}(E / N=56.6 \mathrm{Td})$ crosses over that for $200 \mathrm{~Pa}$. Considering that the drift velocity in $\mathrm{CF}_{4}$ at $B=0$ has the negative differential dependence on $E / N$ at 20-60 Td (Bordage et al 1999), it is suggested that $\boldsymbol{W}$ has such a dependence also in $\boldsymbol{E} \times \boldsymbol{B}$ fields.

\section{Conclusions}

We calculated the electron drift velocity vector $\boldsymbol{W}$ in $\mathrm{F}_{2}$ and $\mathrm{CF}_{4}$ in crossed electric and magnetic fields $(\boldsymbol{E} \times \boldsymbol{B}$ fields). The dependence of $\boldsymbol{W}$ on the reduced electric and magnetic fields, $E / N$ and $B / N$, was depicted as the vector loci of $\boldsymbol{W}$. The behaviour of $\boldsymbol{W}$ was characterized through a comparison with constant-collision-frequency (CCF) models. The vector loci of the CCF model are right semicircles under an electronconservative condition, and its analytical expression was extended to the case in the presence of ionization and attachment. The tendency of $\boldsymbol{W}$ in $\mathrm{F}_{2}$ is close to the CCF

models. In contrast, the vector loci in $\mathrm{CF}_{4}$ has flattened shapes. A kind of negative differential conductivity was indicated in $\mathrm{CF}_{4}$ in $\boldsymbol{E} \times \boldsymbol{B}$ fields, as well as for the case of $B=0$.

\section{Acknowledgments}

Authors H S and Y S wish to thank Professor R M Gilgenbach of The University of Michigan for helpful comments. 


\section{References}

Blevin H A and Haydon S C 1958 Aust. J. Phys. 11 18-34

Bordage M-C, Ségur P, Christophorou L G and Olthoff J K 1999 J. Appl. Phys. 86 3558-66

Bunting K A and Heylen A E D 1971 Int. J. Electron. 31 9-18

Cramer N F 1997 J. Phys. D: Appl. Phys. 30 2573-84

Dincer M S and Gokmen A 1992 J. Phys. D: Appl. Phys. 25 942-4

Dincer M S 1993 J. Phys. D: Appl. Phys. 26 1427-31

Hayashi M and Nimura T 1983 J. Appl. Phys. 54 4879-82

Heylen A E D and Bunting K A 1969 Int. J. Electron. 27 1-12

Heylen A E D and Dargon C L 1973 Int. J. Electron. 35 433-51

Heylen A E D 1980 IEE Proc. 127 221-44

Hopwood J 1992 Plasma Sources Sci. Technol. 1 109-16

Milsom P K 1993 J. Phys. D: Appl. Phys. 26 237-43

Nakamura S, Ventzek P L G and Kitamori K 1999 J. Appl. Phys. 85 2534-9

Nanbu K and Kondo S 1997 Japan. J. Appl. Phys. 36 4808-14

Pekker L 1995 Plasma Sources Sci. Technol. 4 31-5

Shimura N and Makabe T 1993 Appl. Phys. Lett. 62 678-80

Shon C H, Park J S, Kang B K and Lee J K 1999 Japan. J. Appl. Phys. 38 4440-9

Skullerud H R 1968 J. Phys. D: Appl. Phys. 1 1567-8

White R D, Brennan M J and Ness K F 1997 J. Phys. D: Appl. Phys. 30 810-6

White R D, Ness K F, Robson R E and Li B 1999 Phys. Rev. E 60 2231-49 


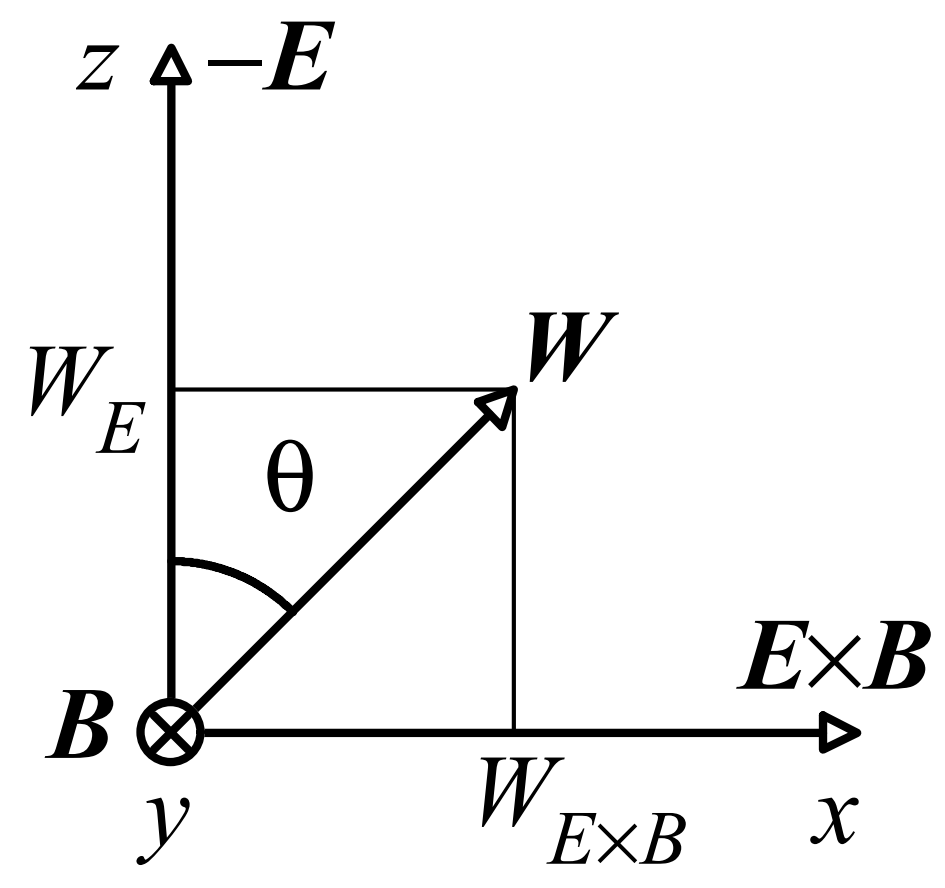

Figure 1. Coordinate system of real space $(x, y, z)$ and the drift velocity components $W_{E}$ and $W_{E \times B} . \theta$ is the Hall deflection angle.

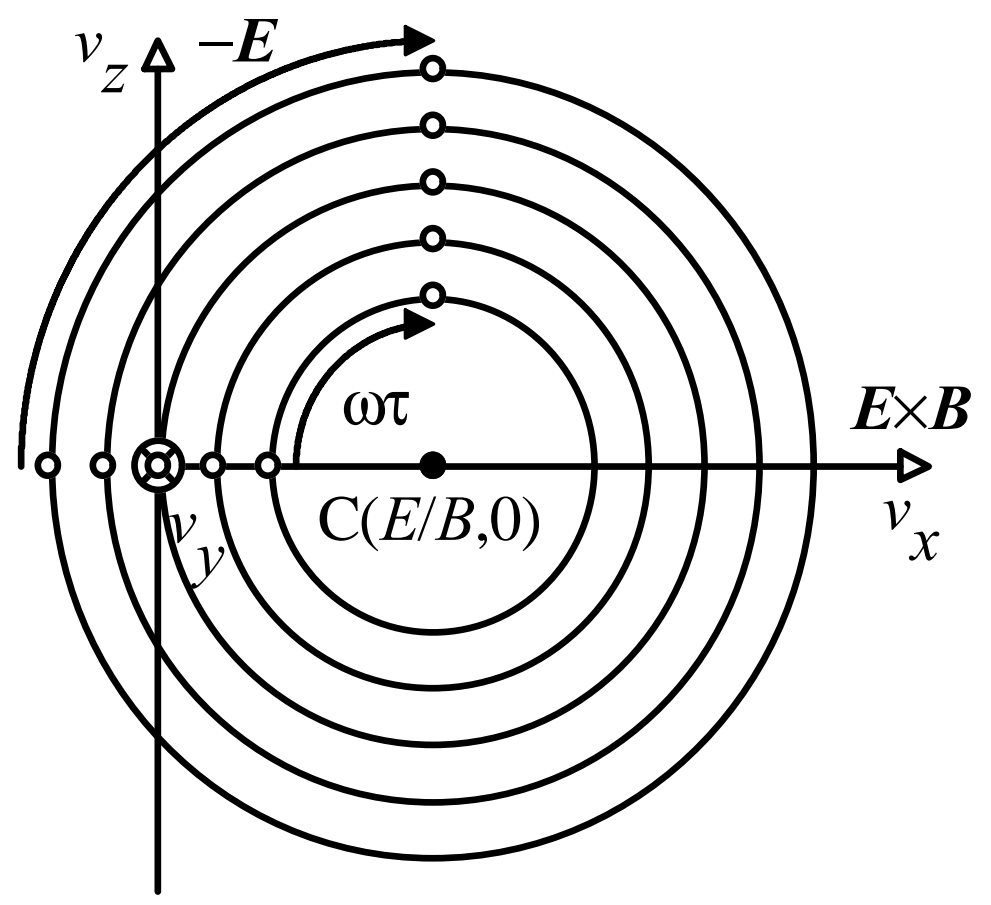

Figure 2. Trajectories of electrons in velocity space. The electrons round on the center $\left(v_{x}, v_{z}\right)=(E / B, 0)$ with a common angular velocity $\omega$ during free flight. 


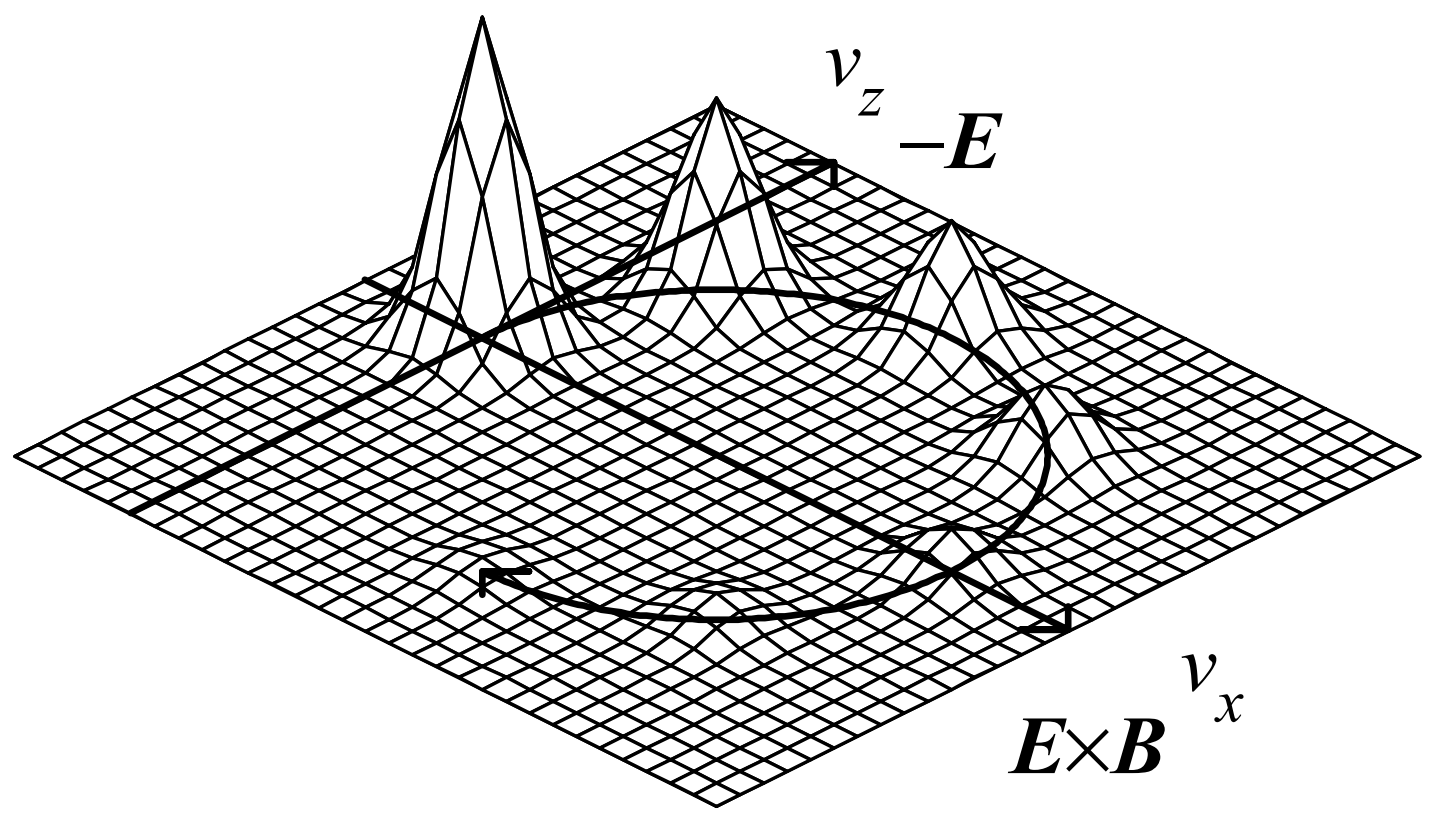

Figure 3. Electron dropout due to collision during collective flight. The electron population decays exponentially keeping the velocity distribution shape isotropic around its center.

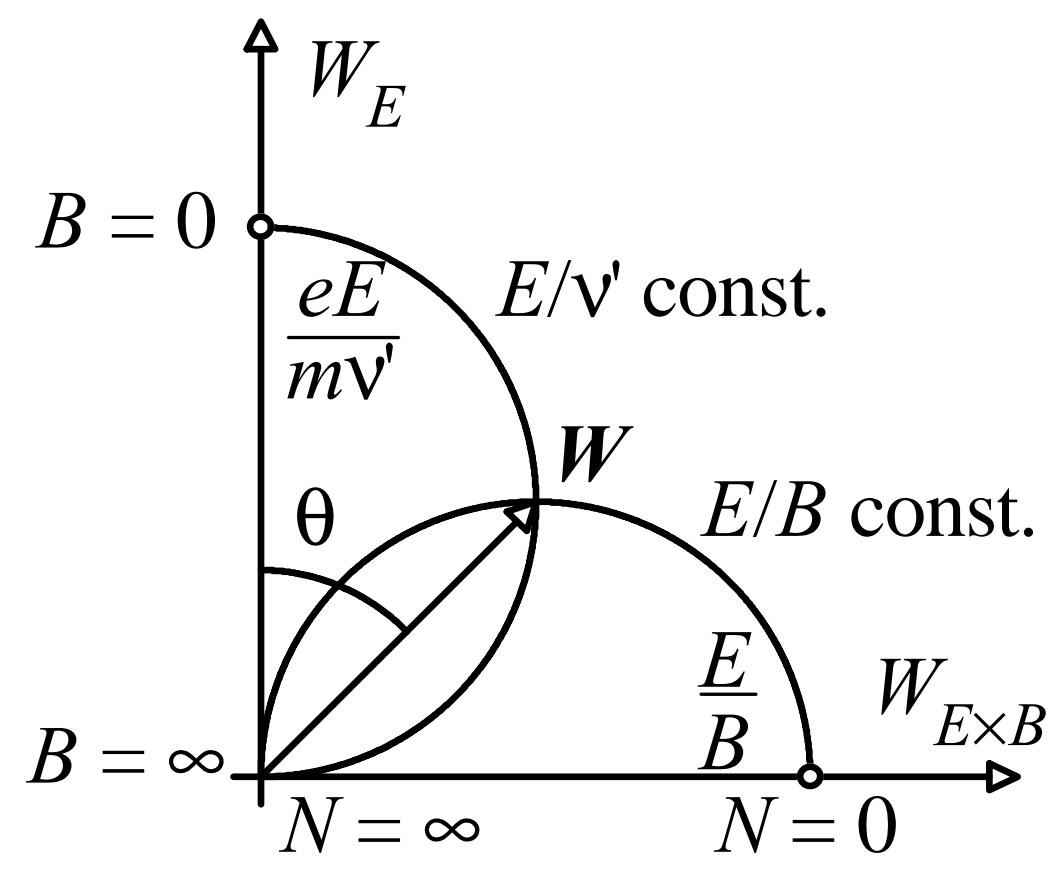

Figure 4. Schematic of the behavior of the drift velocity vector under constant-collisionfrequency condition. 


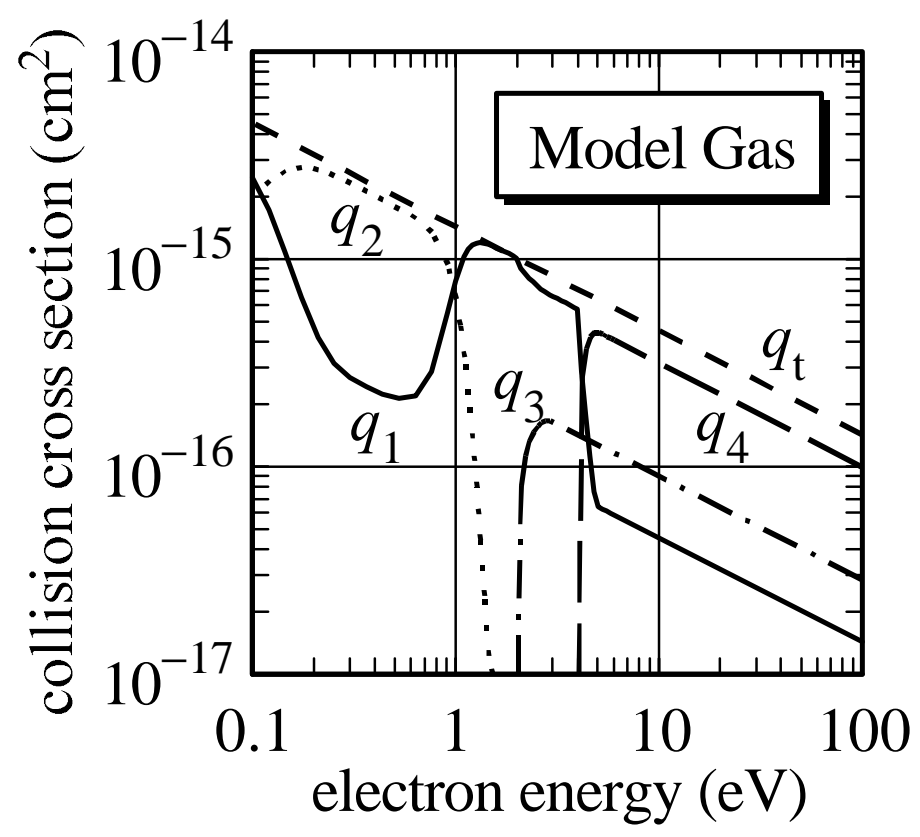

Figure 5. The electron collision cross sections of the constant-collision-frequency model gases. See also table 1 for the assignment of $q_{1}$ through $q_{4}$ to the cross sections of momentum transfer, attachment, excitation and ionization.

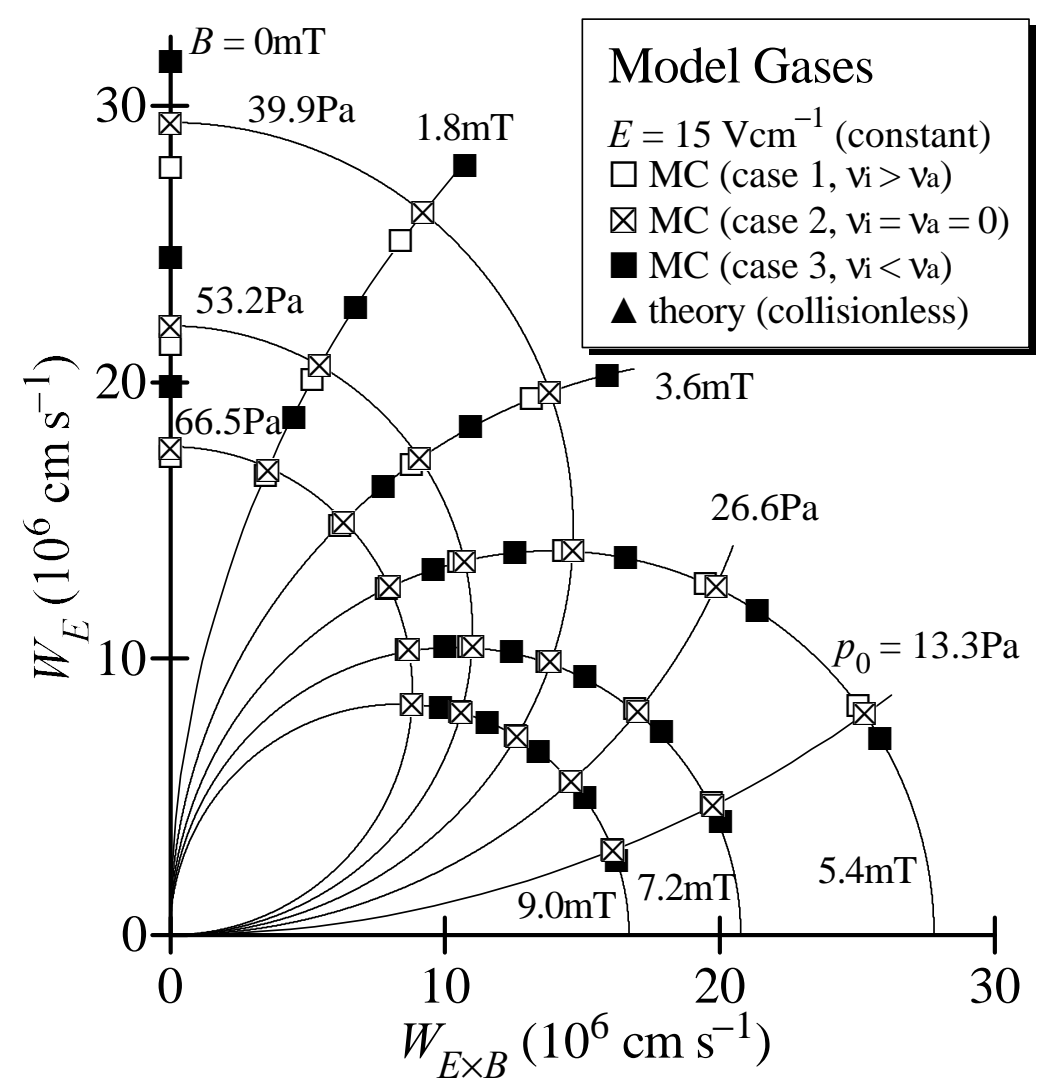

Figure 6. The electron drift velocity vector in the constant-collision-frequency model gases. MC, Monte Carlo; and theory, $W_{E \times B}=E / B$ for collisionless flight. Semicircles depict theoretical prediction under electron-conservative condition. 


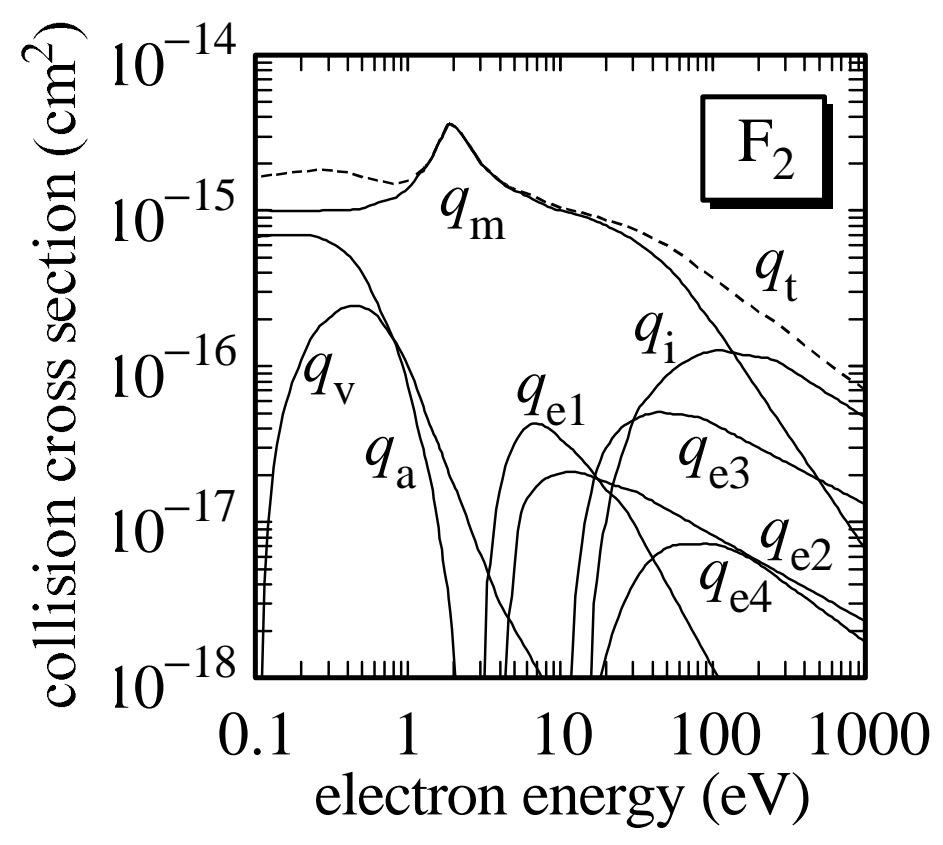

Figure 7. The electron collision cross sections of $\mathrm{F}_{2}$ (Hayashi and Nimura 1983); $q_{\mathrm{m}}$, momentum transfer; $q_{\mathrm{i}}$, ionization; $q_{\mathrm{a}}$, attachment; $q_{\mathrm{v}}$, vibrational excitation; $q_{\mathrm{e} 1}$ through $q_{\mathrm{e} 4}$, electronic excitation; and $q_{\mathrm{t}}$, total.

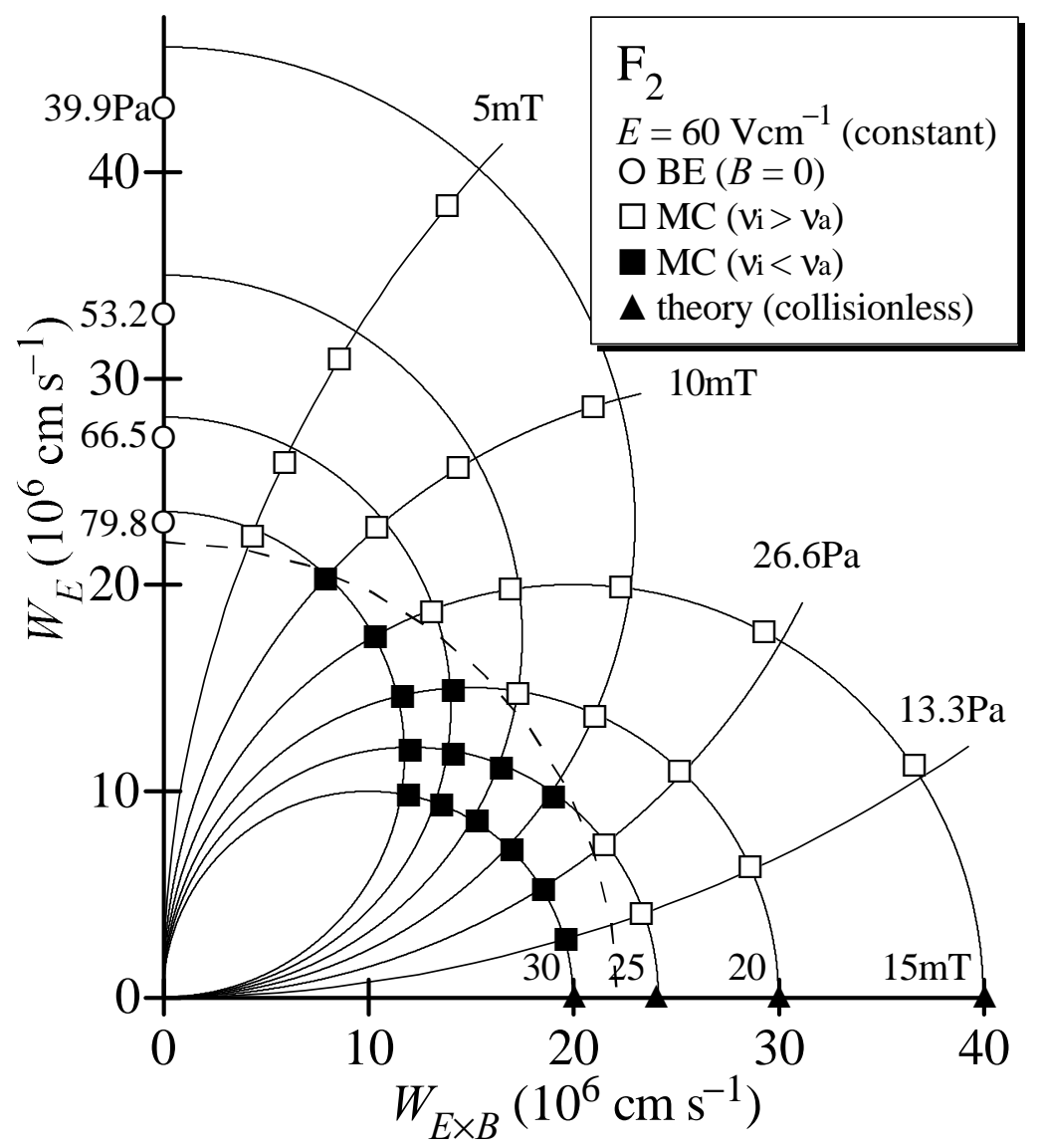

Figure 8. The electron drift velocity vector in $\mathrm{F}_{2}$. BE, Boltzmann equation analysis; $\mathrm{MC}$, Monte Carlo; and theory, $W_{E \times B}=E / B$ for collisionless flight. Semicircles are to guide the eye. 


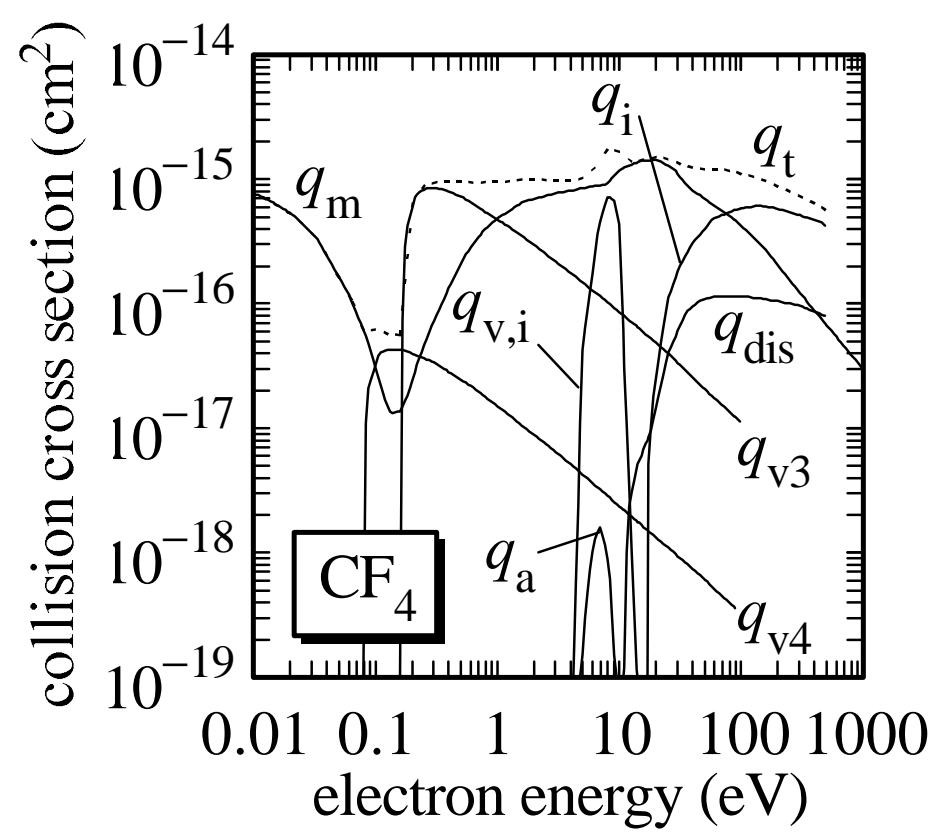

Figure 9. The electron collision cross sections of $\mathrm{CF}_{4}$ (Bordage et al 1999); $q_{\mathrm{m}}$, momentum transfer; $q_{\mathrm{i}}$, ionization; $q_{\mathrm{dis}}$, dissociation; $q_{\mathrm{a}}$, attachment; $q_{\mathrm{v}, \mathrm{i}}$, indirect vibrational excitation; $q_{\mathrm{v} 3}$ and $q_{\mathrm{v} 4}$, vibrational excitation to modes 3 and 4 ; and $q_{\mathrm{t}}$, total.

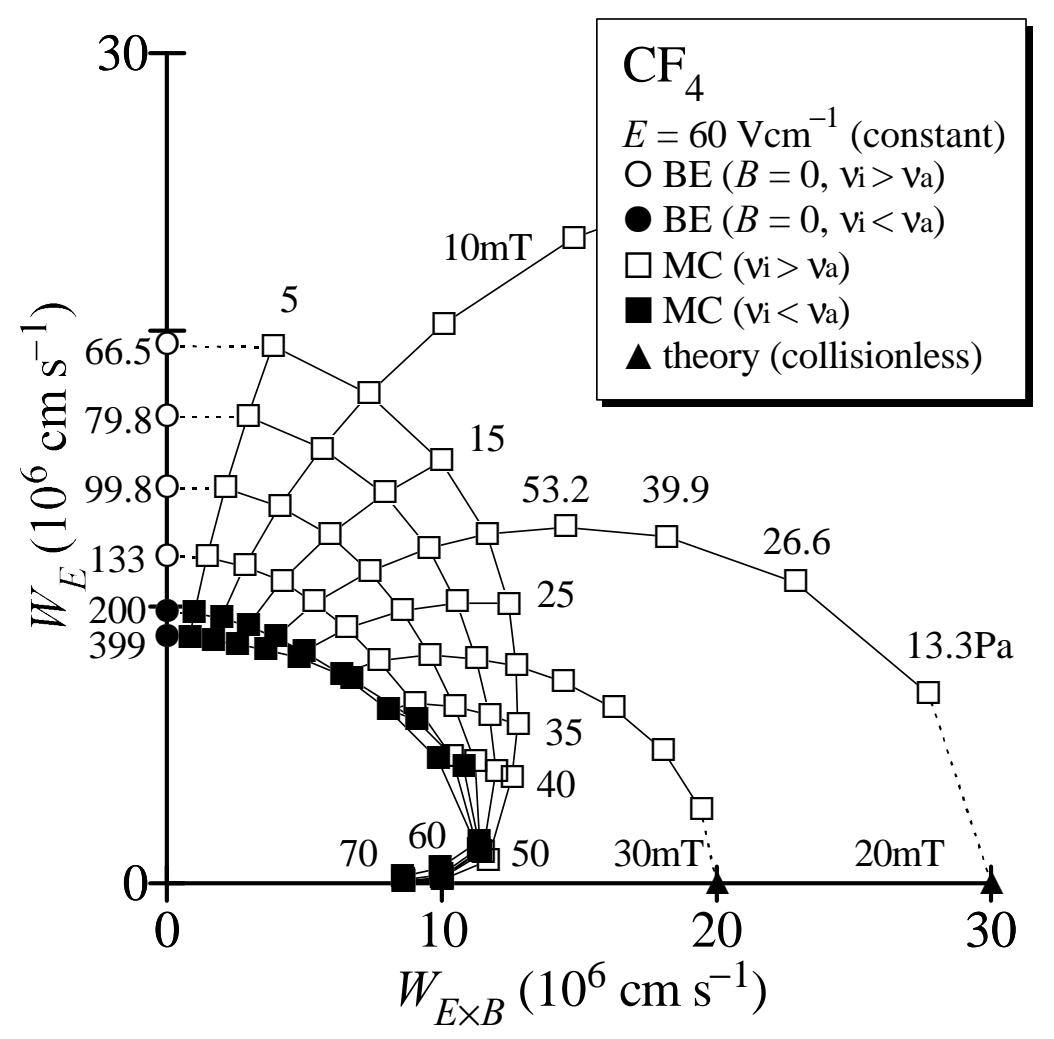

Figure 10. The electron drift velocity vector in $\mathrm{CF}_{4}$. BE, Boltzmann equation analysis; $\mathrm{MC}$, Monte Carlo; and theory, $W_{E \times B}=E / B$ for collisionless flight. 